Revista de Matemática: Teoría y Aplicaciones 2002 9(2) : 31-38

CIMPA - UCR - CCSS ISSN: 1409-2433

\title{
SOME ORNSTEIN-UHLENBECK POTENTIALS FOR THE ONE-DIMENSIONAL SCHRÖDINGER OPERATOR PART II: POSITION-DEPENDENT DRIFT
}

\author{
Santiago Cambronero V.*
}

Received: 17 Apr 2002

\begin{abstract}
We consider the Schrödinger operator on the unit circle, whose potential is an Ornstein - Uhlenbeck type process, with drift depending on its position. We describe the distribution of the periodic groundstate, based on the circular brownian motion measure. The results exposed here, have been mentioned, but not proved, in [7].
\end{abstract}

Keywords: Schrödinger Operator, Ornstein - Uhlenbeck Process, Periodic Groundstate, Circular Brownian Motion.

\section{Resumen}

Se considera el operador de Schrödinger en el círculo unitario, con un potencial de tipo Ornstein - Uhlenbeck, cuyo factor tendencial depende de la posición. Se describe la distribución del primer valor propio periódico, usando el movimiento browniano circular. Los resultados expuestos aquí, han sido mencionados, pero no demostrados, en [7].

Palabras clave: Operador de Schrödinger, proceso de Ornstein - Uhlenbeck, estado periódico, movimiento browniano circular.

Mathematics Subject Classification: 60H25, 60J65, 65J15.

\section{Introduction}

The standard Ornstein-Uhlenbeck process is the diffusion $q$ satisfying the stochastic differential equation

$$
d q=d b-m q d t
$$

\footnotetext{
${ }^{*}$ CIMPA, Escuela de Matemática, Universidad de Costa Rica, 2060 San José, Costa Rica. E-Mail: scambro@emate.ucr.ac.cr
} 
where $m$ is a constant, and $b$ is a standard Brownian motion. It is explicity given by ${ }^{1}$

$$
q(t)=q(0) e^{-m t}+e^{-m t} \int_{0}^{t} e^{m \tau} d b_{\tau}
$$

where the process $\int_{0}^{t} e^{m \tau} d b_{\tau}$ can be written as $W\left(\frac{e^{2 m t}-1}{2 m}\right)$, for some Brownian motion $W$ starting at 0 . This allows the computation of the transition density for $q$, obtaining in particular that $\int p(1 ; a, a) d a=\left(1-e^{-m}\right)^{-1}<\infty$. The process $q$ can be made periodic by conditioning it to have $q(1)=q(0)$. The resulting finite-dimensional distributions are:

$$
P\left[q_{t_{0}} \in d a_{0}, \ldots, q_{t_{n-1}} \in d a_{n-1}\right]=\left(1-e^{-m}\right) \prod_{i=1}^{n} p\left(t_{i}-t_{i-1} ; a_{i-1}, a_{i}\right) d a_{0} \ldots d a_{n-1},
$$

where $0=t_{0}<t_{1}<\ldots<t_{n}=1$, and $a_{n}=a_{0}$.

The coordinate process in $\mathcal{C}\left(S^{1}\right)$ is a periodic Ornstein - Uhlenbeck process under the probability measure $d Q_{*}$ on $\mathcal{C}\left(S^{1}\right)$, defined by

$$
\begin{aligned}
E_{*}[\phi] & =\left.\left(1-e^{-m}\right) \int\left\{\frac{\partial}{\partial x} E_{a}^{o u}[\phi(q), q(1) \leq x]\right\}\right|_{x=a} d a \\
& =\left(1-e^{-m}\right) \int E_{a}^{o u}[\phi(q) \mid q(1)=a] p(1 ; a, a) d a .
\end{aligned}
$$

Moreover, by the Cameron-Martin formula one can get the following relation between $d Q_{*}$ and the circular Brownian motion measure $d \mu_{*}$ on $\mathcal{C}\left(S^{1}\right)$ :

$$
\int \phi(q) d Q_{*}=E_{*}[\phi]=\sqrt{\frac{2}{\pi}} \sinh \left(\frac{m}{2}\right) \int \phi(q) e^{-\frac{m^{2}}{2} \int q^{2}} d \mu_{*} .
$$

The details can be found in [5].

Remark: Taking $\phi \equiv 1$, this gives the corollary:

$$
\int e^{-\frac{m^{2}}{2} \int_{0}^{1} q^{2}} d \mu_{*}=\sqrt{\frac{\pi}{2}}\left[\sinh \left(\frac{m}{2}\right)\right]^{-1}
$$

With respect to the probability $P_{0}$, induced by $\mu_{*}$ on $H:=\left\{q: \int_{0}^{1} q=0\right\}$, we get the following identity:

$$
E^{0}\left[e^{-\frac{m^{2}}{2} \int_{0}^{1} q^{2}}\right]=\frac{m}{2 \sinh \left(\frac{m}{2}\right)}
$$

The whole argument can be applied to the more general case $d q=-m(q) d t+d b$, for any reasonable drift $m$. This is done in the next section. The case of $m=m(t) q$ is treated in $[5]$.

\section{The equation. What is known}

We consider Hill's equation:

$$
-y^{\prime \prime}+q y=\lambda y
$$

the potential $q$ being the periodic Ornstein-Ulenbeck process. We think of $q$ as being any element in the set $\Omega=\mathcal{C}\left(S^{1}\right)$, and impose in $\Omega$ the probability measure $Q_{*}$. Let $\lambda_{0}(q)$ denote the periodic groundstate of $(2)$, with potential $q$. In [5] we prove the following:

\footnotetext{
${ }^{1}$ See $[9]$, or $[6]$.
} 
Theorem 1 Under the transformation $Q=\lambda+p^{\prime}+p^{2}$, the probability measure $Q_{*}$, restricted to $\left[\lambda_{0} \geq \lambda\right]$, is transformed into the measure $d P_{0} d \alpha$, according to the following: If $\phi$ is a bounded measurable function on $\Omega$, then

$$
\int_{\left[\lambda_{0} \geq \lambda\right]} \phi d Q_{*}=C \int_{H} \int_{I\left(p^{\prime}\right)}^{\infty} \phi\left(\lambda+p^{\prime}+p^{2}\right) e^{-\frac{m^{2}}{2} \int\left(\lambda+p^{\prime}+p^{2}\right)^{2}} G\left(\alpha, p^{\prime}\right) d \alpha d P_{0}\left(p^{\prime}\right),
$$

with $C=\frac{4}{\sqrt{2 \pi}} \sinh \left(\frac{m}{2}\right), p=\alpha+\int_{0}^{t} p^{\prime}, I\left(p^{\prime}\right)=-\int_{0}^{1} \int_{0}^{t} p^{\prime}$, and

$$
G\left(\alpha, p^{\prime}\right)=\exp \left[\int_{0}^{1}\left(p^{\prime 3}-2 p^{2} p^{2}\right) d t\right] \sinh \left(\int_{0}^{1} p\right) .
$$

Corollary 1 The distribution of $\lambda_{0}$ under $Q_{*}$ is given by

$$
Q_{*}\left[\lambda_{0} \geq \lambda\right]=C \int_{H} \int_{I\left(p^{\prime}\right)}^{\infty} e^{-\frac{m^{2}}{2} \int\left(\lambda+p^{\prime}+p^{2}\right)^{2}} G\left(\alpha, p^{\prime}\right) d \alpha d P_{0}\left(p^{\prime}\right)
$$

\section{The new potentials}

Let us consider the diffusion $q$ solving

$$
d q=-m(q) d t+d b
$$

where $b$ is a standard Brownian motion under a probability measure $P$, and $m$ is an odd function, with $m(q)>0$ for $q>0$ (to avoid explossion). Thus $q$ has associated the infinitesimal generator

$$
\frac{1}{2} \frac{d^{2}}{d q^{2}}-m(q) \frac{d}{d q}
$$

under $P$.

We denote by $\hat{Q}$ the probability measure under which $q$ is Brownian motion. Notice that, for $q(t)>0$,

$$
q(t)=b(t)-b\left(t_{0}\right)-\int_{t_{0}}^{t} m\left(q_{s}\right) d s \leq b(t)-b\left(t_{0}\right),
$$

with $t_{0}=\operatorname{máx}\{s \leq t: Q(s)=0\}$. Similarly, for $q(t)<0$,

$$
q(t)=b(t)-b\left(t_{0}\right)-\int_{t_{0}}^{t} m\left(q_{s}\right) d s \geq b(t)-b\left(t_{0}\right),
$$

and therefore, in any case

$$
|q(t)| \leq 2 \operatorname{máx}_{0 \leq s \leq t}|b(t)|, \text { for all } t \geq 0 .
$$

In particular $q(t)$ is defined for all $t \geq 0$.

Let $t_{N}=\inf \{t \geq 0:|q(t)|=N\}$. On $\left[t_{N} \geq t\right]$ we have

$$
\operatorname{máx}_{0 \leq s \leq t}|q(s)| \leq N \text {. }
$$


Since $m(q)$ is bounded on $[-N, N]$, we can apply Cameron-Martin to the process $q\left(t \wedge t_{N}\right)$, i.e. for any bounded $\phi$,

$$
E_{a}^{P}\left\{\phi(q) \chi_{\left[t_{N} \geq t\right]}\right\}=\hat{E}_{a}\left\{\phi(q) Z(t) \chi_{\left[t_{N} \geq t\right]}\right\},
$$

with

$$
Z(t):=\exp \left[-\int_{0}^{t} m\left(q_{s}\right) d q_{s}-\frac{1}{2} \int_{0}^{t} m^{2}\left(q_{s}\right) d s\right] .
$$

Since $t_{N} \uparrow \infty$, taking $\phi \equiv 1$ and using dominated convergence gives

$$
\hat{E}_{a}\{Z(t)\}=\lim _{N \rightarrow \infty} \hat{E}_{a}\left\{Z(t) \chi_{\left[t_{N} \geq t\right]}\right\}=\lim _{N \rightarrow \infty} E_{a}^{P}\left\{\chi_{\left[t_{N} \geq t\right]}\right\}=1 .
$$

Thus, we can apply Cameron-Martin to the process $q(t)$ itself.

Now we define a probability measure $Q_{*}$ (under which $q$ will be periodic) by setting

$$
\begin{aligned}
E^{*}[\phi] & =\left.C \int\left\{\frac{\partial}{\partial x} E_{a}^{P}[\phi(q), q(1) \leq x]\right\}\right|_{x=a} d a \\
& =\left.C \int\left\{\frac{\partial}{\partial x} \hat{E}_{a}[\phi(q) Z(1), q(1) \leq x]\right\}\right|_{x=a} d a \\
& =C \int E^{00}\left[\phi(q+a) e^{-\frac{1}{2} \int_{0}^{1} F\left(a+q_{s}\right) d s}\right] \frac{d a}{\sqrt{2 \pi}} \\
& =\frac{C}{\sqrt{2 \pi}} \int \phi(q) e^{-\frac{1}{2} \int_{0}^{1} F\left(q_{s}\right) d s} d \mu_{*},
\end{aligned}
$$

where $\mu_{*}$ is the circular Brownian motion measure, $F(q)=-m^{\prime}(q)+m^{2}(q)$ and the constant $C$ is to be determined. We want to have

$$
\int_{-\infty}^{\infty} E^{00}\left[e^{-\frac{1}{2} \int_{0}^{1} F\left(a+q_{s}\right) d s}\right] d a<\infty
$$

By Jensen's inequality and Fubini's theorem, it is enough that

$$
\int_{-\infty}^{\infty} e^{-\frac{1}{2} F(y)} d y<\infty, \quad \text { ie. } \int_{-\infty}^{\infty} e^{\frac{1}{2}\left(m^{\prime}-m^{2}\right)}<\infty
$$

If that is the case, then

$$
d Q_{*}=C_{0} e^{-\frac{1}{2} \int_{0}^{1} F\left(q_{s}\right) d s} d \mu_{*}
$$

with

$$
C_{0}=\left[\int e^{-\frac{1}{2} \int_{0}^{1} F\left(q_{s}\right) d s} d \mu_{*}\right]^{-1} .
$$

\section{Distribution of $\lambda_{0}(Q)$}

We want to find the distribution of $\lambda_{0}(q)$ with respect to the probability measure $Q_{*}$. For this, we first express $d Q_{*}$ in terms of $d \alpha d P_{0}\left(p^{\prime}\right)$. By the Cameron - Martin relation obtained above, it is enough to find an expression for $d \mu_{*}$ in terms of $d \alpha d P_{0}\left(p^{\prime}\right)$. More precisely, we consider the transformation

$$
Q=\lambda+p^{\prime}+p^{2},
$$


but instead of $Q_{*}$, we use $\mu_{*}$. The calculations are done in [5], obtaining

$$
d \mu_{*}=2 \exp \left[\int_{0}^{1}\left(\left(p^{\prime}\right)^{3}-2 p^{2}\left(p^{\prime}\right)^{2}\right) d t\right] \sinh \left(\int_{0}^{1} p\right) d \alpha d P_{0} .
$$

In other words, for $\phi$ satisfying $\int|\phi| d \mu_{*}<\infty$,

$$
\int_{\left[\lambda_{0} \geq \lambda\right]} \phi(q) d \mu_{*}=2 \int_{H} \int_{I\left(p^{\prime}\right)}^{\infty} \phi\left(\lambda+p^{\prime}+p^{2}\right) G\left(\alpha, p^{\prime}\right) d \alpha d P_{0}
$$

with $G\left(\alpha, p^{\prime}\right)$ given by

$$
G\left(\alpha, p^{\prime}\right)=\exp \left[\int_{0}^{1}\left(\left(p^{\prime}\right)^{3}-2 p^{2}\left(p^{\prime}\right)^{2}\right) d t\right] \sinh \left(\int_{0}^{1} p\right) .
$$

It follows that

$$
\int_{\left[\lambda_{0} \geq \lambda\right]} \phi(q) d Q_{*}=2 C_{0} \int_{H} \int_{I\left(p^{\prime}\right)}^{\infty} \hat{\phi}(\lambda) e^{-\frac{1}{2} \int_{0}^{1} \hat{F} d s} G\left(\alpha, p^{\prime}\right) d \alpha d \mu_{*}
$$

where we have written $\hat{\phi}(\lambda)$ for $\phi\left(\lambda+p^{\prime}+p^{2}\right)$, and similarly for $\widehat{F}$. In particular, the following theorem follows.

Theorem 2 Let $m(q)$ be an odd function such that $m(q)>0$ for $q>0$, and satisfying (4). If $Q_{*}$ is given by (5), then the distribution function $Q_{*}\left[\lambda_{0} \geq \lambda\right]$ of $\lambda_{0}(Q)$ under $Q_{*}$ is given by

$$
Q_{*}\left[\lambda_{0} \geq \lambda\right]=2 C_{0} \int_{H} \int_{I\left(p^{\prime}\right)}^{\infty} \exp \left[-\frac{1}{2} \int_{0}^{1} F\left(\lambda+p_{s}^{\prime}+p_{s}^{2}\right) d s\right] G\left(\alpha, p^{\prime}\right) d \alpha d P_{0} .
$$

\section{Joint Distribution}

Consider the periodic diffusion coming from the infinitesimal operator

$$
\frac{1}{2} \frac{d^{2}}{d q^{2}}-m(q) \frac{d}{d q}
$$

under the probability measure $Q_{*}$ defined by

$$
d Q_{*}=C_{0} \exp \left[-\frac{1}{2} \int_{0}^{1} F\left(Q_{s}\right) d s\right] d P_{*},
$$

as explained in the last section, with $F=-m^{\prime}+m^{2}$ and

$$
C_{0}^{-1}=\int \exp \left[-\frac{1}{2} \int_{0}^{1} F\left(Q_{s}\right) d s\right] d P_{*}
$$

We consider the transformation $(Q, a) \leftrightarrow\left(p^{\prime}, \alpha, \lambda\right)$ given by

$$
Q=\lambda+p^{\prime}+p^{2}, \quad a=\int_{0}^{1}\left(\alpha+\int_{0}^{t} p^{\prime}\right) d t .
$$


To get the relation between $d P_{*} d a$ and $d P_{0}\left(p^{\prime}\right) d \alpha d \lambda$, it is enough to take care of the Jacobian. But this is done in the same way as for white noise (see [7]). It follows that, if $\int|\phi(Q, a)| d P_{*} d a<\infty$, then $^{2}$

$$
\int \phi d P_{*} d a=\int_{H} \int\left[\int \phi\left(\lambda+p^{\prime}+p^{2}, \int p\right) d \lambda\right] E\left(\alpha, p^{\prime}\right) e^{-\int p} d \alpha d P_{0},
$$

with $p=\alpha+\int_{0}^{t} p^{\prime}$ and

$$
E\left(\alpha, p^{\prime}\right)=\exp \left[\int_{0}^{1}\left(p^{\prime 3}-2 p^{2} p^{2}\right)\right] A(p) .
$$

Here we use the notation

$$
A(p)=\int_{0}^{1} e^{-2 \int_{0}^{x} p} d x \int_{0}^{1} e^{2 \int_{0}^{x} p} d x
$$

Notice that $\alpha=I\left(p^{\prime}\right)$ if and only if $\int_{0}^{1} p=0$.

By (6) and (7), if $\int|\varphi(Q, a)| d Q_{*} d a<\infty$, then

$$
\int \varphi d Q_{*} d a=C_{0} \int_{H} \int\left[\int \hat{\varphi}(\lambda) e^{-\frac{1}{2} \int \hat{F}(\lambda)} d \lambda\right] e^{-\int p} E\left(\alpha, p^{\prime}\right) d \alpha d P_{0}
$$

where $\hat{\varphi}(\lambda)$ denotes $\varphi\left(\lambda+p^{\prime}+p^{2}, \int p\right)$ and $\hat{F}(\lambda)$ denotes $F\left(\lambda+p_{s}^{\prime}+p_{s}^{2}\right)$. In particular, for the classical Ornstein-Uhlenbeck case,

$$
\int \varphi d \hat{Q} d a=C \int_{H} \int\left[\int \hat{\varphi} e^{-\frac{m^{2}}{2} \int\left(\lambda+p^{\prime}+p^{2}\right)^{2}} d \lambda\right] e^{-\int p} E\left(\alpha, p^{\prime}\right) d \alpha d P_{0},
$$

with $C=\sqrt{\frac{2}{\pi}} \sinh \left(\frac{m}{2}\right)$.

\section{Induced Measure and Density of $\lambda_{0}(Q)$}

The results of the last section will be used to find the density of $\lambda_{0}(Q)$ under $P_{*}$ and $Q_{*}$. We first notice that the choice $\phi(Q)=\varphi(Q) \chi_{[0 \leq t \leq h]}$ in $(7)$ gives

$$
\int \varphi d P_{*}=\int_{H} \frac{1}{h} \int^{+h}\left[\int_{-\infty}^{\infty} \varphi\left(\lambda+p^{\prime}+p^{2}\right) d \lambda\right] e^{-\int_{0}^{1} p} E\left(\alpha, p^{\prime}\right) d \alpha d P_{0}
$$

and, as $h \rightarrow 0$,

$$
\int \varphi(Q) d P_{*}=\int_{H}\left(\int_{-\infty}^{\infty} \varphi\left(\lambda+p^{\prime}+p^{2}\right) d \lambda\right) E\left(p^{\prime}\right) d P_{0}
$$

Here, $E\left(p^{\prime}\right):=E\left(I\left(p^{\prime}\right), p^{\prime}\right)$ and $p=I\left(p^{\prime}\right)+\int_{0}^{t} p^{\prime}$.

Since $\int_{0}^{1} Q$ is Lebesgue - distributed under $P_{*}$, taking $\left.\varphi=\chi_{\left[0 \leq \int\right.} Q \leq 1\right]$ produces

$$
\int_{H} E\left(p^{\prime}\right) d P_{0}=1
$$

\footnotetext{
${ }^{2}$ In the following $d P_{0}$ will denote $d P_{0}\left(p^{\prime}\right)$.
} 
i.e.,

$$
\int_{H} \exp \left[\int_{0}^{1}\left(p^{\prime 3}-2 p^{2} p^{\prime 2}\right)\right]\left(\int_{0}^{1} e^{-2 \int_{0}^{x} p} d x \int_{0}^{1} e^{2 \int_{0}^{x} p} d x\right) d P_{0}=1 .
$$

Since $\lambda_{0}\left(\lambda+p^{\prime}+p^{2}\right)=\lambda$ when $\int p=0$, we also have

$$
\int \varphi(Q) \chi_{\left[\mu \leq \lambda_{0} \leq \rho\right]} d P_{*}=\int_{H}\left(\int_{\mu}^{\rho} \varphi\left(\lambda+p^{\prime}+p^{2}\right) d \lambda\right) E\left(p^{\prime}\right) d P_{0}
$$

for $-\infty \leq \mu<\rho \leq \infty$. This easily implies the following expression for the measure $P_{*}^{\mu}$ induced by $P_{*}$ on $\left[\lambda_{0}=\mu\right]$ :

$$
\int_{\left[\lambda_{0}=\mu\right]} \varphi(Q) d P_{*}^{\mu}=\int_{H} \varphi\left(\mu+p^{\prime}+p^{2}\right) E\left(p^{\prime}\right) d P_{0} .
$$

Because of (9), taking $\varphi \equiv 1$ implies:

Proposition. $\lambda_{0}(Q)$ is Lebesgue-distributed under $P_{*}$. The corresponding formulas for $Q_{*}$ are

$$
\int \varphi(Q): d Q_{*}=C_{0} \int_{H}\left[\int \hat{\varphi}(\lambda) e^{-\frac{1}{2} \int \hat{F}(\lambda)} d \lambda\right] E\left(p^{\prime}\right) d P_{0}
$$

and

$$
\int_{\left[\lambda_{0}=\mu\right]} \varphi(Q) d Q_{*}^{\mu}=C_{0} \int_{H} \hat{\varphi}(\mu) e^{-\frac{1}{2} \int \hat{F}(\mu)} E\left(p^{\prime}\right) d P_{0} .
$$

In particular, the density of $\lambda_{0}$ under $Q_{*}$ is

$$
f(\mu)=C_{0} \int_{H} e^{-\frac{1}{2} \int F\left(\mu+p^{\prime}+p^{2}\right)} E\left(p^{\prime}\right) d P_{0} .
$$

For the classical Ornstein-Uhlenbeck case,

$$
\int_{\left[\lambda_{0}=\mu\right]} \varphi(Q) d \hat{Q}_{\mu}=\sqrt{\frac{2}{\pi}} \sinh \left(\frac{m}{2}\right) \int_{H} \hat{\varphi}(\mu) e^{-\frac{m^{2}}{2} \int_{0}^{1}\left(\mu+p^{\prime}+p^{2}\right)^{2}} E\left(p^{\prime}\right) d P_{0}
$$

and

$$
f(\mu)=\sqrt{\frac{2}{\pi}} \sinh \left(\frac{m}{2}\right) \int_{H} e^{-\frac{m^{2}}{2} \int_{0}^{1}\left(\mu+p^{\prime}+p^{2}\right)^{2}} E\left(p^{\prime}\right) d P_{0} .
$$

\section{Some identities}

Now we deduce a series of identities involving the measures $Q_{*}, P_{*}$ and $P_{0}$, based on the results of previous sections.

Let us start by applying (7) to $\phi(Q, a)=\psi(Q) e^{-a^{2} / 2}$. The result is

$$
\sqrt{2 \pi} \int \psi(Q) d P_{*}=\int_{H} \int_{\mathbb{R}}\left[\int_{\mathbb{R}} \hat{\psi}\left(\lambda, p^{\prime}\right) d \lambda\right] e^{-\frac{1}{2}\left(\int p\right)^{2}-\int p} E\left(\alpha, p^{\prime}\right) d \alpha d P_{0},
$$

which for $\psi(Q)=\chi_{\left[0 \leq \int Q \leq 1\right]}$ gives

$$
\int_{H} \int_{-\infty}^{\infty} e^{-\frac{1}{2}\left(\int p\right)^{2}-\int p} E\left(\alpha, p^{\prime}\right) d \alpha d P_{0}=\sqrt{2 \pi} .
$$


More generally,

$$
\int_{H} \int_{-\infty}^{\infty} \varphi\left(\int p\right) e^{-\int p} E\left(\alpha, p^{\prime}\right) d \alpha d P_{0}=\int_{-\infty}^{\infty} \varphi(x) d x .
$$

Compare with (9). Next, take $\varphi=\varphi\left(\int Q\right)$ in (10) to obtain

$$
\int \varphi\left(\int p\right) d Q_{*}=C_{0} \int_{H}\left[\int \varphi\left(\lambda+\int p^{2}\right) e^{-\frac{1}{2} \int \hat{F}(\lambda)} d \lambda\right] E\left(p^{\prime}\right) d P_{0} .
$$

In the Ornstein-Uhlenbeck, with $\varphi \equiv 1$, this gives

$$
\int_{H} e^{-\frac{m^{2}}{2}\left[\int p^{4}+\int\left|p^{\prime}\right|^{2}-\left(\int p^{2}\right)^{2}\right]} E\left(p^{\prime}\right) d P_{0}=\frac{m}{2 \sinh \left(\frac{m}{2}\right)},
$$

and (11) reduces to

$$
\int \varphi\left(\int Q\right) d \hat{Q}=\frac{m}{\sqrt{2 \pi}} \int_{-\infty}^{\infty} \varphi(x) e^{-\frac{m^{2}}{2} x^{2}} d x
$$

i.e., $\int_{0}^{1} Q \sim\left(0, m^{-2}\right)$ under $\hat{Q}$. In a similar way, for $\varphi(Q, a)=\psi\left(\int Q\right) \varphi(a)$ in (8),

$$
\int_{H} \int_{\mathbb{R}} e^{-\frac{m^{2}}{2}\left[\int p^{4}+\int p^{\prime 2}-\left(\int p^{2}\right)^{2}\right]} \varphi\left(\int p\right) e^{-\int p} E\left(\alpha, p^{\prime}\right) d \alpha d P_{0}=\frac{m}{2 \sinh \left(\frac{m}{2}\right)} \int_{\mathbb{R}} \varphi .
$$

These identities seem to be very useful as a tool in solving some open problems on asymptotic behaviour of the density as $|\lambda| \rightarrow \infty$.

\section{Referencias}

[1] Cambronero, S. (1996) The Distribution of the Ground State of Hill's Equation with Random Potential. Ph.D. Thesis. Courant Institute of Mathematical Sciences. New York University.

[2] McKean, H.; Cambronero, S. (1995) "Valores Propios de Dirichlet asociados a la ecuación de Hill con potencial de ruido blanco", Revista de Matemática: Teoría y Aplicaciones 2(2): 1-7.

[3] Cambronero, S. (1997) "La ecuación de Hill y el movimiento browniano circular", V Enc. Centroam.de Invest. en Matemática, Liberia, Universidad de Costa Rica: 133-140.

[4] Cambronero, S. (1996) "La ecuación de Hill con potencial irregular", Revista de Matemática: Teoría y Aplicaciones 3(1): 25-34.

[5] Cambronero, S. (1997) "Algunos potenciales del tipo Ornstein - Uhlenbeck para el operador de Schrödinger", Revista de Matemática: Teoría y Aplicaciones 4(1): 61-77.

[6] Itô, K.; Mckean, H.P. (1974) Diffusion Processes and their Sample Paths. 2nd ed. Springer.

[7] McKean, H.P.; Cambronero, S. (1999) "The ground state eigenvalue of Hill's equation with white noise potential", Comm. in Pure and Applied Mathematics, LII: 1277-1294.

[8] Fukushima, M.; Nakao, S. (1977) "On spectra of the Schrödinger operator with a white noise potential", Z. Wahrsch. Verb. Geb. 37: 267-274.

[9] Karatzas, I.; Shreve, S.E. (1991) Brownian Motion and Stochastic Calculus. 2nd ed., Springer.

[10] McKean, H.P. (1994) "A limit law for the ground gtate of Hill's equation", J. of Stat. Phys. 74(5/6): 1227-1232. 